\title{
Interphase cytogenetic analysis with centromeric probes for chromosomes 1, 2, 6, 10 , and 17 in 11 tumors from a patient with bilateral renal oncocytosis
}

\author{
Paolo Cossu-Rocca ${ }^{1,2}$, John N Eble ${ }^{1}$, Shaobo Zhang ${ }^{1}$, Stephen M Bonsib ${ }^{3}$, \\ Guido Martignoni ${ }^{4}$, Matteo Brunelli ${ }^{2}$ and Liang Cheng ${ }^{1,5}$ \\ ${ }^{1}$ Department of Pathology and Laboratory Medicine, Indiana University, Indianapolis, IN, USA; \\ ${ }^{2}$ Dipartimento di Patologia, Universitá di Sassari, Sassari, Italy; ${ }^{3}$ Department of Pathology and Laboratory \\ Medicine, Louisiana State University Health Science Center, Shreveport, LA, USA; ${ }^{4}$ Dipartimento di \\ Patologia, Universitá di Verona, Verona, Italy and ${ }^{5}$ Department of Urology, Indiana University, \\ Indianapolis, IN, USA
}

\begin{abstract}
Renal oncocytosis is characterized by the presence of multiple tumors with oncocytic features, often associated with small clusters of tubule-like structures with oncocytic change. The morphologic features of the oncocytic nodules encompass a spectrum of appearances, with patterns typical of renal oncocytoma or classic chromophobe renal cell carcinoma, as well as 'hybrid' tumors with features resembling both oncocytoma and chromophobe renal cell carcinoma. We utilized interphase cytogenetic methods to study 11 tumors from the kidneys of a 45-year-old woman. The tumors included morphologically classical oncocytomas and 'hybrid' tumors with features reminiscent of chromophobe carcinoma. The kidneys also showed foci of oncocytic change in renal tubules. Fluorescence in situ hybridization was performed with centromeric probes for chromosomes 1, 2, 6, 10, and 17 in each of the 11 tumors to determine whether or not there were losses of the chromosomes that are most frequently lost in chromophobe renal cell carcinomas. Neoplastic nuclei from each tumor were evaluated for the number of hybridization signals and scored according to the percentage of nuclei with one, two, and three or more signals. The normal renal parenchyma surrounding the tumors was used as control tissue. All 11 tumors from this patient with renal oncocytosis showed no loss of any of the chromosomes 1, 2, 6, 10, or 17, a pattern identical to that found in normal control tissues. These observations weigh against the concept that hybrid tumors of oncocytosis are closely related to chromophobe renal cell carcinoma.
\end{abstract}

Modern Pathology (2008) 21, 498-504; doi:10.1038/modpathol.2008.16; published online 1 February 2008

Keywords: kidney; renal oncocytosis; fluorescence in situ hybridization

Renal oncocytosis is characterized by the presence of multiple oncocytic tumors, often associated with oncocytic changes in renal tubules. A similar condition occurs in patients with Birt-Hogg-Dubé syndrome. ${ }^{1-3}$ Oncocytosis was originally described in 1982 by Warfel and Eble ${ }^{1}$ in a 67-year-old woman with more than 200 renal oncocytomas, which they called 'renal oncocytomatosis.' Tickoo et $a l^{2}$ in 1999 described 14 cases, 6 of which had bilateral renal

Correspondence: Dr L Cheng, MD, Department of Pathology and Laboratory Medicine, Indiana University School of Medicine, 350 West 11th Street, Clarian Pathology Laboratory Room 4010, Indianapolis, IN 46202, USA.

E-mail: lcheng@iupui.edu

Received 19 March 2007; revised 18 July 2007; accepted 8 August 2007; published online 1 February 2008 involvement. All the kidneys had numerous oncocytic nodules, most of which closely resembled renal oncocytoma or, less often, chromophobe renal cell carcinoma. Some of the nodules were considered to be 'hybrid' because they had morphologic features of both oncocytoma and chromophobe renal cell carcinoma. Associated findings were diffuse oncocytic changes in non-neoplastic renal tubules, or single or clustered oncocytic cells infiltrating non-neoplastic tubules. Tickoo et al proposed the term 'renal oncocytosis' to encompass the entire spectrum of lesions. Several papers have described cases with multiple and bilateral renal oncocytomas, but only a few appear to have been renal oncocytosis. ${ }^{4-7}$

Due to the association of different morphologic patterns, renal oncocytosis raises the question of the possibility of a close relationship between renal 
oncocytoma and chromophobe renal cell carcinoma. Renal oncocytosis may also pose a diagnostic problem in surgical pathology, because of the different prognoses of oncocytoma and chromophobe renal cell carcinoma. ${ }^{8,9}$ Numerous features have been proposed to accurately distinguish oncocytoma from chromophobe renal cell carcinoma, including histochemical, immunohistochemical, and ultrastructural findings, but all of them have shown some limitations. Since chromophobe carcinomas differ from oncocytomas in having multiple chromosomal losses, the identification of specific chromosomal losses is an additional tool for distinguishing these tumors. ${ }^{10}$

We undertook to analyze 11 tumors from a patient with bilateral renal oncocytosis using fluorescence in situ hybridization (FISH) with centromeric probes for the chromosomes most frequently lost in chromophobe renal cell carcinomas (chromosomes 1, 2, 6,10 , and 17) to test the hypothesis that the tumors of renal oncocytosis are related to chromophobe renal cell carcinoma.

\section{Clinical history and pathology}

The 45-year-old white woman with multiple bilateral renal masses was initially admitted to the Indiana University Hospital for wedge resection of multiple tumors from the left kidney. There was no history of Birt-Hogg-Dubé syndrome. Four tumors were resected: one from the medial aspect of the kidney and three from the lateral aspect. The medial tumor was a $20 \times 20 \times 10 \mathrm{~mm}$, firm, well-circumscribed, light-brown nodule with a $3 \mathrm{~mm}$ central stellate area of fibrosis. The lateral tumors were $10 \times 10,8 \times 4 \times 5$, and $10 \times 10 \times 8 \mathrm{~mm}$. All were light brown and one had a focus of hemorrhage in it. Microscopic examination of the tumors showed them to be composed of cells with abundant finely granular eosinophilic cytoplasm and mainly spherical, but occasionally somewhat irregular, nuclei with easily visible nucleoli. The cells were arranged in a compact array with delicate vasculature, punctuated by scattered small cysts. In some cells, perinuclear cytoplasmic clearing was present. In addition to the principal tumors, there were $1 \mathrm{~mm}$ nodules in the adjacent renal tissue associated with the left hilar tumor and the left lateral tumor 3 . These small tumors were histologically identical to the larger tumors. Hale's colloidal iron stain was performed on three tumors and each showed a blue band of cytoplasm at the apical end of the cells. Immunohistochemistry with antibody to cytokeratin 7 showed positive reactions in the cytoplasm of scattered single cells and small clusters of cells. Renal capsule and renal sinus were not involved by any of the tumors.

In December 2004, the patient underwent right radical nephrectomy. The right kidney contained 20 grossly visible tumors, concentrated in the midkidney and superior pole, with cut surfaces ranging from pale-tan to brown with hemorrhage. The tumors ranged in size from 1 to $55 \mathrm{~mm}$. Neither invasion of perinephric fat nor renal vein invasion was grossly discernible. The adrenal gland was normal. Microscopic examination of these tumors showed appearances identical to those described above for the tumors from the left kidney. The grossly normal renal parenchyma between the tumors showed widespread oncocytic changes in renal tubules and scattered microscopic nodules with irregular contours. Hale's colloidal iron stain showed a mixed pattern, with areas of negative tumor cells alternating with areas in which the neoplastic cells displayed bands of positively reacting cytoplasm at the apical ends of the cells. Immunohistochemistry showed negative reactions for CD10 and vimentin, and positive reactions in the cytoplasm of scattered cells and small clusters of cells with antibody to cytokeratin 7. Microscopically, the renal capsule, renal vein, and adrenal gland were not involved by the tumors. A small renal sinus vein contained intraluminal tumor. Two renal sinus lymph nodes and two paracaval lymph nodes were negative for metastasis.

Although some of the tumors showed morphology overlapping between oncocytoma and chromophobe renal cell carcinoma, none of the lesions from either kidney showed a histologic appearance typical of chromophobe renal cell carcinoma.

\section{Materials and methods}

\section{Tissue Samples}

Sections $4 \mu \mathrm{m}$ thick were obtained from paraffin blocks from the four tumors from the left kidney and from seven of the tumors from the right kidney. The tumors studied ranged in size from 2 to $55 \mathrm{~mm}$. As much as possible, specimens also included normal renal parenchyma surrounding the tumors. Sections stained with hematoxylin and eosin showed patterns typical of oncocytoma in nine of the tumors, whereas two (tumors 10 and 11 in Table 1) had morphology overlapping between oncocytoma and chromophobe renal cell carcinoma (Figure 1).

\section{Fluorescence In Situ Hybridization Analysis}

FISH was performed as described previously ${ }^{10-17}$ with centromeric $\alpha$-satellite DNA probes for chromosome 1 (centromeric enumeration probe (CEP) 1, spectrum orange), chromosome 2 (CEP 2, spectrum orange), chromosome 6 (CEP 6, spectrum green), chromosome 10 (CEP 10, spectrum green), and chromosome 17 (CEP 17, spectrum orange) (Figure 2). All the probes were from Vysis (Vysis, Downers Grove, IL, USA). The slides were examined using a Zeiss Axioplan 2 microscope (Zeiss, Göttingen, Germany) with the following filters: SP-100 DAPI, FITC MF-101 for spectrum green (CEP 6 and 10) and Gold 31003 for 
Table 1 Percentages of nuclei with hybridization signals in distinct tumors from renal oncocytosis

\begin{tabular}{|c|c|c|c|c|c|c|}
\hline \multirow[t]{2}{*}{ Tumor } & \multicolumn{3}{|c|}{ Chromosome 1} & \multicolumn{3}{|c|}{ Chromosome 2} \\
\hline & 1 signal (\%) & 2 signals (\%) & $\geq 3$ signals (\%) & 1 signal (\%) & 2 signals (\%) & $\geq 3$ signals $(\%)$ \\
\hline 1 & 34 & 65 & 2 & 36 & 62 & 2 \\
\hline 2 & 36 & 63 & 2 & 35 & 63 & 2 \\
\hline 3 & 35 & 63 & 2 & 36 & 62 & 2 \\
\hline 4 & 35 & 63 & 2 & 37 & 61 & 2 \\
\hline 5 & 36 & 62 & 2 & 37 & 61 & 2 \\
\hline 6 & 37 & 61 & 2 & 35 & 64 & 1 \\
\hline 7 & 35 & 64 & 2 & 32 & 66 & 2 \\
\hline 8 & 36 & 63 & 2 & 36 & 63 & 2 \\
\hline 9 & 37 & 61 & 2 & 32 & 66 & 2 \\
\hline 10 & 38 & 61 & 1 & 37 & 61 & 2 \\
\hline 11 & 36 & 62 & 2 & 36 & 62 & 2 \\
\hline \multirow[t]{2}{*}{ Tumor } & \multicolumn{3}{|c|}{ Chromosome 6} & \multicolumn{3}{|c|}{ Chromosome 10} \\
\hline & 1 signal (\%) & 2 signals (\%) & $\geq 3$ signals $(\%)$ & 1 signal (\%) & 2 signals (\%) & $\geq 3$ signals $(\%)$ \\
\hline 1 & 33 & 65 & 2 & 37 & 61 & 2 \\
\hline 2 & 35 & 63 & 2 & 36 & 62 & 2 \\
\hline 3 & 33 & 67 & 1 & 36 & 63 & 1 \\
\hline 4 & 36 & 62 & 2 & 35 & 63 & 2 \\
\hline 5 & 34 & 64 & 2 & 37 & 62 & 1 \\
\hline 6 & 35 & 64 & 2 & 37 & 61 & 2 \\
\hline 7 & 33 & 65 & 2 & 36 & 62 & 2 \\
\hline 8 & 33 & 65 & 2 & 36 & 63 & 1 \\
\hline 9 & 35 & 64 & 1 & 33 & 65 & 2 \\
\hline 10 & 34 & 65 & 1 & 36 & 62 & 2 \\
\hline 11 & 35 & 64 & 2 & 36 & 62 & 2 \\
\hline \multirow[t]{2}{*}{ Tumor } & \multicolumn{3}{|c|}{ Chromosome 17} & & & \\
\hline & 1 signal (\%) & 2 signals (\%) & $\geq 3$ signals $(\%)$ & & & \\
\hline 1 & 37 & 62 & 2 & & & \\
\hline 2 & 36 & 62 & 2 & & & \\
\hline 3 & 36 & 62 & 2 & & & \\
\hline 4 & 36 & 62 & 2 & & & \\
\hline 5 & 35 & 63 & 2 & & & \\
\hline 6 & 36 & 62 & 2 & & & \\
\hline 7 & 36 & 63 & 2 & & & \\
\hline 8 & 34 & 65 & 2 & & & \\
\hline 9 & 33 & 66 & 1 & & & \\
\hline 10 & 36 & 63 & 2 & & & \\
\hline 11 & 36 & 62 & 2 & & & \\
\hline
\end{tabular}

spectrum orange (CEP 1, 2, and 17) from Chroma (Chroma, Brattleboro, VT, USA). The images were acquired with a CCD camera and analyzed with MetaSystem Isis software (MetaSystem, Belmont, MA, USA). Five sequential focus stacks with $0.4 \mu \mathrm{m}$ intervals were acquired and then integrated into a single image to reduce thickness-related artifacts.

For each tumor, 100-200 nuclei were scored for signals from centromeric probes under the fluorescence microscope with $\times 1000$ magnification. Fifty nuclei from non-neoplastic renal parenchyma were also scored as control. Signals from solitary nuclei were preferentially counted, but signals from adjacent, non-overlapping nuclei were occasionally included in the counts. Two signals of the same size in close proximity, not connected by a link, were counted as two separate signals. A diffuse signal was regarded as a signal if it was contiguous and within an acceptable boundary. Two small signals connected by a visible link were counted as one signal. Overlapping nuclei and nuclei with uncertain signals were not included in the counts. Definition of chromosomal gain was based on the Gaussian model and related to the data from the normal renal parenchyma controls. A tumor with a percentage of nuclei with single signals beyond the mean +3 s.d. of the normal parenchymal control was considered to have lost that chromosome.

A case of chromophobe renal cell carcinoma was used as control (Figure 2f). 

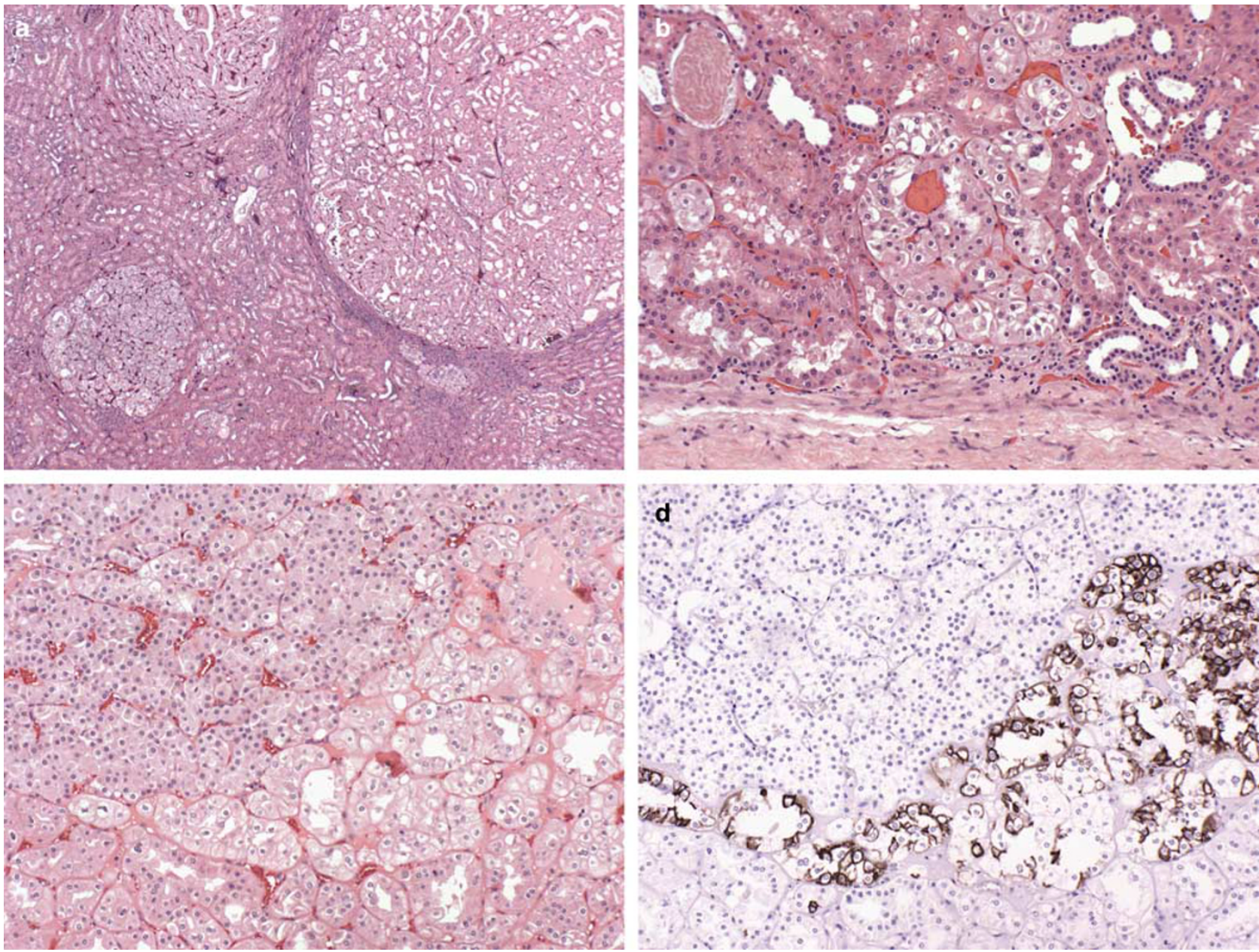

Figure 1 Renal oncocytosis. (a) Multiple oncocytic nodules are appreciable in a low-power magnification field. (b) Oncocytic changes in non-neoplastic tubules. (c) Tumor with hybrid morphology showing areas of oncocytoma-like and chromophobe carcinoma-like proliferations. (d) Immunohistochemistry for cytokeratin 7 (same field) demonstrated a focal positivity in the chromophobe carcinoma-like areas.

\section{Results}

The distribution of signals in non-neoplastic tissues for each probe was assessed in renal parenchyma adjacent to the tumors and in sections from blocks of non-neoplastic renal parenchyma from the right nephrectomy. Section thickness and tissue processing were the same for normal and neoplastic tissues. For chromosomes 1, 2, 6, 10, and 17, the mean percentages of nuclei with two hybridization signals were $64,65,66,63$, and $64 \%$, respectively. The mean percentages of nuclei with single signals were $35,34,33,36$, and $35 \%$, respectively. The mean percentage of nuclei with three or more signals was $1 \%$ for each of the chromosomal probes. The standard deviations of the mean numbers of nuclei with single signals for chromosomes $1,2,6,10$, and 17 were $1.3,1.8,1,0.4$, and $0.7 \%$, respectively. Chromosomal loss would be recognized in a tumor if the mean percentage of nuclei with single signals was greater than the mean +3 s.d. for that chromosome in non-neoplastic control tissue. For chromosome 1, this was $35 \%+(3 \times 1.3 \%)=38.9 \%$. For chromosome 2 , this was $34 \%+(3 \times 1.8 \%)=39.4 \%$. For chromosome 6 , this was $33 \%+(3 \times 1 \%)=36 \%$. For chromosome 10 , this was $36 \%+(3 \times 0.4 \%)=37.2 \%$. For chromosome 17 , this was $35 \%+(3 \times 0.7 \%)=37.1 \%$.

The results for the tumors are presented in Table 1. For chromosome 1, the percentages of nuclei with single signals ranged from 33.5 to $37.6 \%$ (mean $=35.7 \%$, s.d. $=1.29$ ); none showed loss of chromosome 1. For chromosome 2, the percentages of nuclei with single signals ranged from 32.1 to $37.1 \%$ (mean $=35.4 \%$, s.d. $=1.75)$; none showed loss of chromosome 2. For chromosome 6, the percentages of nuclei with single signals ranged from 32.5 to $35.6 \%$ (mean $=34.1 \%$, s.d. $=1.01)$; none showed loss of chromosome 6 . For chromosome 10, the percentages of nuclei with single signals ranged from 33.3 to $36.7 \%$ (mean $=35.9 \%$, s.d. $=0.98)$; none showed loss of chromosome 10. For chromosome 17, the percentages of nuclei with single signals ranged from 33.0 to $36.5 \%$ (mean $=35.4 \%$, s.d. $=1.13)$; none showed loss of chromosome 17. 
a

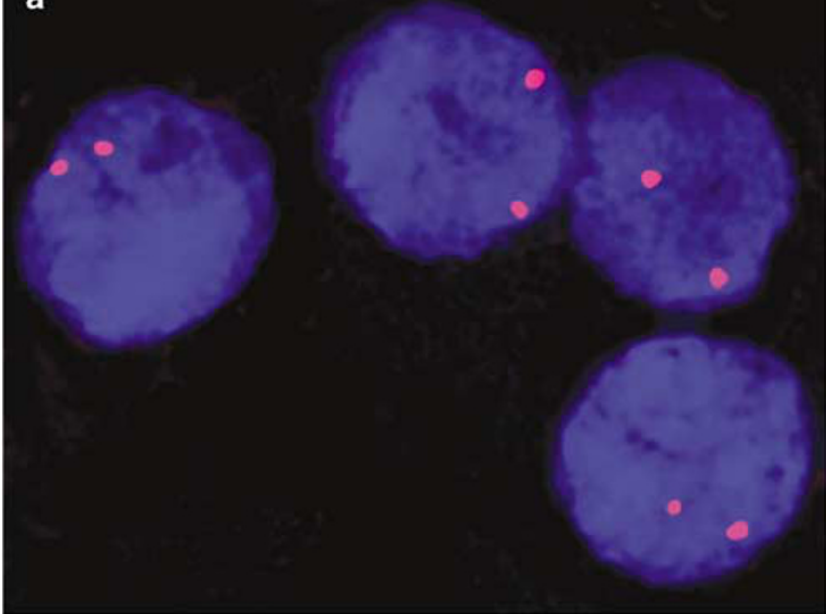

\section{C}

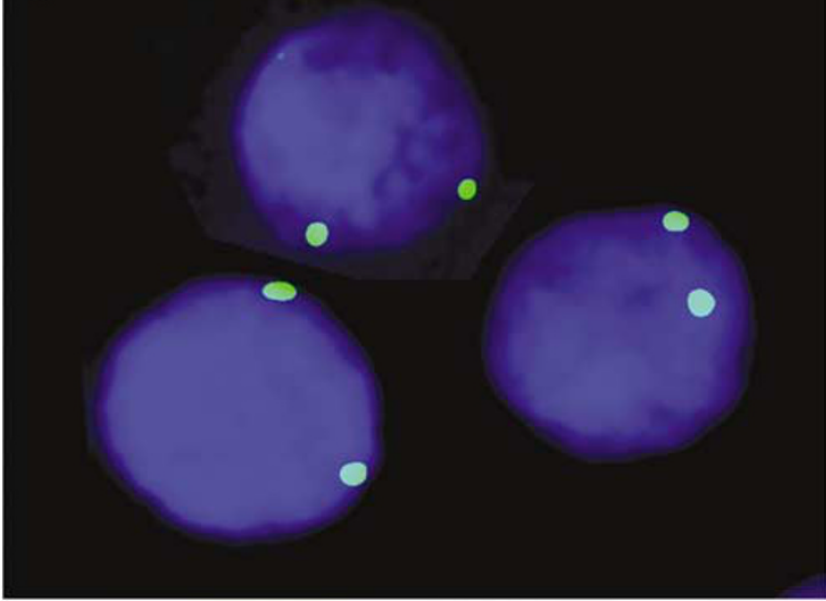

e

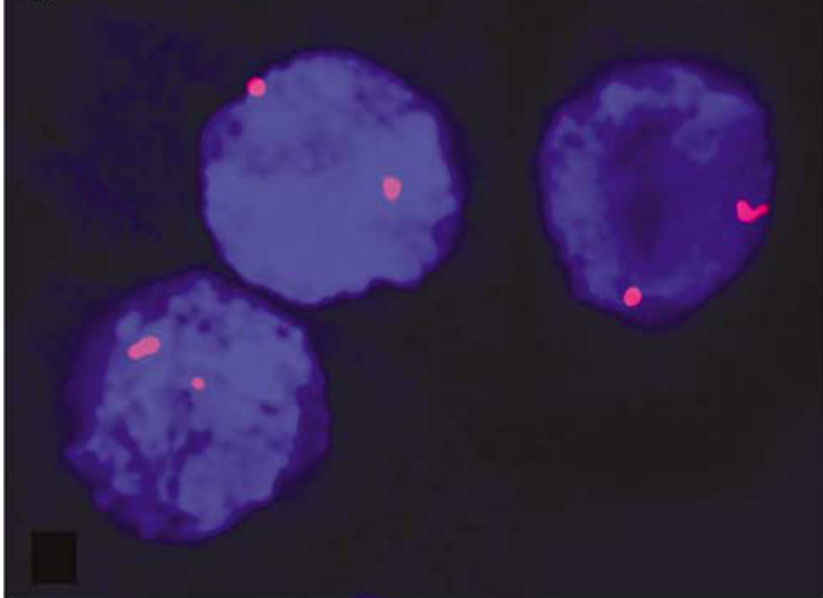

b

d

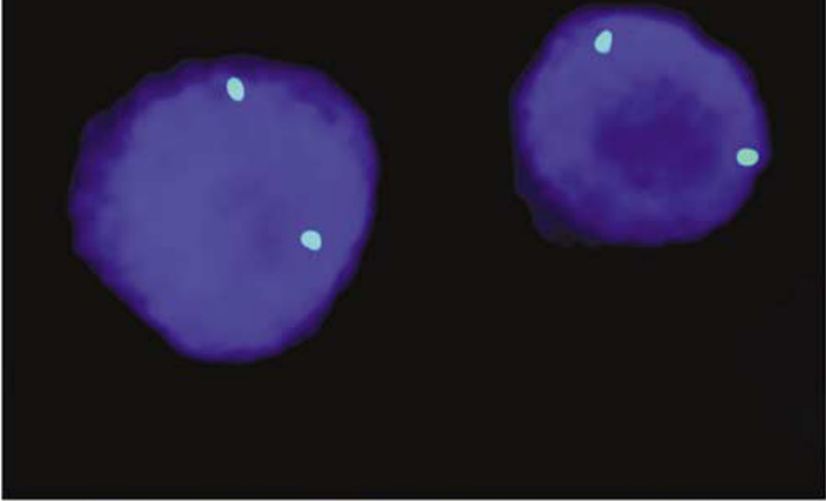

\section{f}

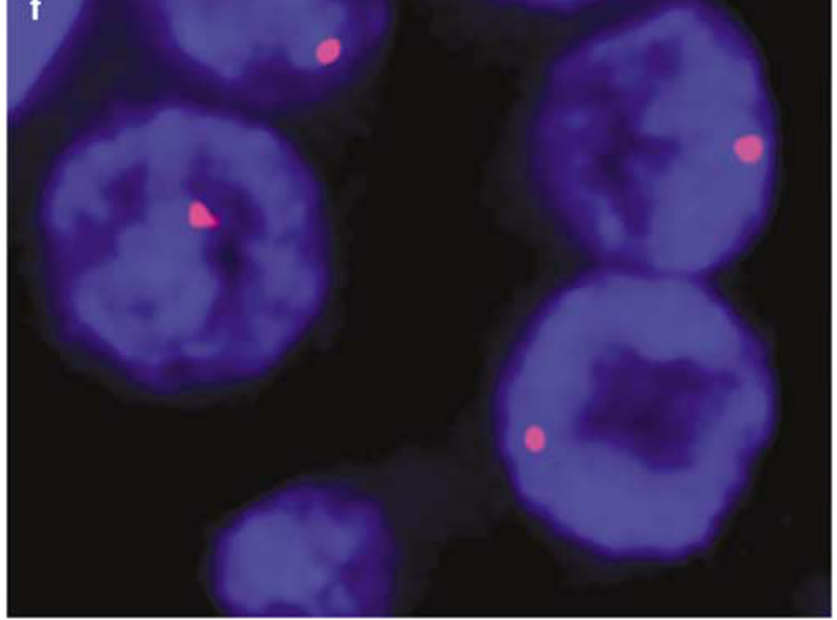

Figure 2 Fluorescence in situ hybridization: two hybridization signals were detected in the nuclei of a great majority of cells in all the tumors. (a) Centromeric probe for chromosome 1 (spectrum orange). (b) Centromeric probe for chromosome 2 (spectrum orange). (c) Centromeric probe for chromosome 6 (spectrum green). (d) Centromeric probe for chromosome 10 (spectrum green). (e) Centromeric probe for chromosome 17 (spectrum orange). (f) By contrast, chromophobe renal cell carcinoma showing nuclei with single fluorescent signal for chromosome 1, indicating a chromosome loss. 
The results for nuclei with three or more signals are shown in Table 1 . These were tightly clustered for each of the chromosomes and none of the tumors showed any gains of chromosomes.

The control chromophobe case showed loss of chromosomes 1, 2, 6, 10, and 17 .

\section{Discussion}

We report the interphase cytogenetic analyses by FISH of 11 tumors from a case of bilateral renal oncocytosis, not related to Birt-Hogg-Dubé syndrome, finding that none of the tumors showed any losses of chromosomes 1, 2, 6, 10, or 17 (the chromosomes most frequently lost in chromophobe renal cell carcinomas). ${ }^{10}$ Reports of genetic analyses of renal oncocytosis are few. Leroy et $\mathrm{al}^{7}$ reported a normal karyotype in two tumors histologically resembling oncocytomas in a case of bilateral renal oncocytosis associated with renal failure. Chen et al ${ }^{5}$ reported a probable deletion of chromosome 20 in 2 of 20 metaphases in a tumor from a 12-year-old girl with multiple tumors resembling renal oncocytomas or with features of both oncocytoma and chromophobe renal cell carcinoma. Using classical cytogenetics and microsatellite analysis for loss of heterozygosity (LOH), Al-Saleem et $a l^{18}$ studied five tumors and a focus of oncocytic change in renal tubules from a case of bilateral renal oncocytosis. A hybrid tumor showed losses of chromosomes 1, 14, 21, and Y and microsatellite analyses showed LOH for chromosomal arms $1 \mathrm{p}, 14 \mathrm{q}$, and 21q. ${ }^{18}$ Cytogenetically, a tumor resembling oncocytoma had a normal karyotype. Microsatellite analysis of two tumors resembling oncocytomas from the opposite kidney showed LOH only for chromosome 1p in one and no LOH in the other. Microsatellite analysis showed no LOH in a tumor resembling chromophobe renal cell carcinoma nor in the oncocytic changes in renal tubules. ${ }^{18}$ In our study, performed with FISH, in 11 different tumors, we found no losses of chromosomes 1,2, 6, 10, and 17. These tumors included those that closely resembled morphologically typical renal oncocytomas and also hybrid tumors with chromophobe-like areas.

Combining the data from Al-Saleem et $a l^{18}$ and the present study, genetic information is available for three hybrid tumors from cases of renal oncocytosis. One showed losses of chromosomes 1, 14, 21, and Y by classical cytogenetic methods, whereas the other two tumors showed no loss of any of chromosomes $1,2,6,10$, or 17 . Thus, none of the three tumors showed loss of any of chromosomes 2, 6, 10, or 17 . Since losses of one or more of these chromosomes are extremely common in chromophobe renal cell carcinomas ${ }^{10}$ the data from these three tumors offer little or no support for the hypothesis that the 'hybrid' tumors of renal oncocytosis are, in some way, intermediate between oncocytoma and chromophobe renal cell carcinoma. 'Hybrid' tumors are common in renal oncocytosis, whereas sporadic renal tumors with hybrid morphologic patterns are, at most, extremely rare, with only six having been reported, none with genetic analysis. ${ }^{19,20}$

Combining the data from Leroy et al, ${ }^{7} \mathrm{Al}-\mathrm{Saleem}$ et $a l,{ }^{18}$ and the present study, 13 tumors resembling oncocytomas from cases of oncocytosis have had some genetic analysis, and loss of $1 p$ demonstrated by microsatellite analysis in one tumor has been the only abnormality detected. A close relationship between renal oncocytoma and chromophobe renal cell carcinoma has been suggested, based on the theory of common origin of these lesions from the intercalated ducts of the distal nephron, the morphologic similarities between renal oncocytoma and the eosinophilic variant of chromophobe renal cell carcinoma, ${ }^{10,21}$ and the coexistence of tumors resembling oncocytoma, chromophobe renal cell carcinoma, and the ones with features of both in the kidneys from patients with renal oncocytosis. ${ }^{2,5,7}$ Since a minority of renal oncocytomas are known to have losses of chromosome 1, the information presently available about the genetics of the tumors resembling oncocytomas is compatible with their being identical to sporadic oncocytomas. However, since it is nearly entirely evidence of lack of abnormalities, it is not specific and should not be taken as strong evidence that the tumors in oncocytosis and sporadic oncocytomas are one and the same. We wish to emphasize that the tumors from patients with Birt-Hogg-Dubé syndrome may have entirely different cytogenetic findings.

In conclusion, we demonstrate that although morphologic and immunophenotypic features resembling oncocytoma and chromophobe renal cell carcinoma are appreciable in the varied oncocytic nodules in renal oncocytosis, genetic studies have failed to find the losses of chromosomes prevalent in chromophobe renal cell carcinomas or the loss of chromosome 1 found in a minority of renal oncocytomas. Thus, renal oncocytosis has not provided genetic evidence that renal oncocytoma and chromophobe renal cell carcinoma are closely related. Further, the morphologically varied tumors of renal oncocytosis may not be genetically similar to the more common sporadic tumors, which some of them resemble. It may be better to diagnose these tumors simply as 'tumors of renal oncocytosis' until there is more clarity about their relationship with other renal tumor and about their prognosis.

\section{References}

1 Warfel KA, Eble JN. Renal oncocytomatosis. J Urol 1982;127:1179-1180.

2 Tickoo SK, Reuter VE, Amin MB, et al. Renal oncocytosis: a morphologic study of fourteen cases. Am J Surg Pathol 1999;23:1094-1101.

3 Pavlovich CP, Walther MM, Eyler RA, et al. Renal tumors in the Birt-Hogg-Dubésyndrome. Am J Surg Pathol 2002;26:1542-1552. 
4 Nagashima Y, Mitsuya T, Shioi KI, et al. Renal oncocytosis. Pathol Int 2005;55:210-215.

5 Chen TS, McNally M, Hulbert W, et al. Renal oncocytosis presenting in childhood: a case report. Int J Surg Pathol 2003;11:325-329.

6 Katz DS, Gharagozloo AM, Peebles TR, et al. Renal oncocytomatosis. Am J Kidney Dis 1996;27:579-582.

7 Leroy X, Lemaitre L, De La Taille A, et al. Bilateral renal oncocytosis with renal failure. Arch Pathol Lab Med 2001;125:683-685.

8 Amin MB, Tamboli P, Javidan J, et al. Prognostic impact of histologic subtyping of adult renal epithelial neoplasms: an experience of 405 cases. Am J Surg Pathol 2002;26:281-291.

9 Cheville JC, Lohse CM, Zincke H, et al. Comparisons of outcome and prognostic features among histologic subtypes of renal cell carcinoma. Am J Surg Pathol 2003;27:612-624.

10 Brunelli M, Eble JN, Zhang S, et al. Eosinophilic and classic chromophobe renal cell carcinomas have similar frequent losses of multiple chromosomes from among chromosomes $1,2,6,10$, and 17 , and this pattern of genetic abnormality is not present in renal oncocytoma. Mod Pathol 2005;18:161-169.

11 Brunelli M, Eble JN, Zhang S, et al. Gains of chromosomes 7, 17, 12, 16, and 20 and loss of Y occur early in the evolution of papillary renal cell neoplasia: a fluorescent in situ hybridization study. Mod Pathol 2003;16:1053-1059.

12 Brunelli M, Eble JN, Zhang S, et al. Metanephric adenoma lacks the gains of chromosomes 7 and 17 and loss of $\mathrm{Y}$ that are typical of papillary renal cell carcinoma and papillary adenoma. Mod Pathol 2003; 16:1060-1063.
13 Cossu-Rocca P, Eble JN, Zhang S, et al. Acquired cystic disease-associated renal tumors: an immunohistochemical and fluorescence in situ hybridization study. Mod Pathol 2006;19:780-787.

14 Cossu-Rocca P, Eble JN, Delahunt B, et al. Renal mucinous tubular and spindle carcinoma lacks the gains of chromosomes 7 and 17 and losses of chromosome $\mathrm{Y}$ that are prevalent in papillary renal cell carcinoma. Mod Pathol 2006;19:488-493.

15 Cossu-Rocca P, Zhang S, Roth LM, et al. Chromosome 12p abnormalities in dysgerminoma of the ovary: a FISH analysis. Mod Pathol 2006;19:611-615.

16 Cheng L, Zhang S, MacLennan GT, et al. Interphase fluorescence in situ hybridization analysis of chromosome $12 \mathrm{p}$ abnormalities is useful for distinguishing epidermoid cysts of the testis from pure mature teratoma. Clin Cancer Res 2006;12:5668-5672.

17 Jones TD, Eble JN, Wang M, et al. Molecular genetic evidence for the independent origin of multifocal papillary tumors in patients with papillary renal cell carcinomas. Clin Cancer Res 2005;11:7226-7233.

18 Al-Saleem T, Cairns P, Dulaimi EA, et al. The genetics of renal oncocytosis: a possible model for neoplastic progression. Cancer Genet Cytogenet 2004;152: 23-28.

19 Noguchi S, Nagashima Y, Shuin T, et al. Renal oncocytoma containing 'chromophobe' cells. Int J Urol 1995;2:279-280.

20 Mai KT, Dhamanaskar P, Belanger E, et al. Hybrid chromophobe renal cell neoplasm. Pathol Res Pract 2005;201:385-389.

21 Polascik TJ, Bostwick DG, Cairns P. Molecular genetics and histopathologic features of adult distal nephron tumors. Urology 2002;60:941-946. 